\title{
Determining the Demands of Disabled People by Artificial Intelligence Methods
}

\author{
Mucahit Karaduman ${ }^{*}$ iD , Ali Karcı ${ }^{2}$ \\ ${ }^{1}$ Arapgir Vocational School, Malatya Turgut Ozal University, Malatya, Turkey \\ ${ }^{2}$ Department of Computer Engineering, Inonu University, Malatya, Turkey \\ (mucahit.karaduman@ozal.edu.tr, ali.karci@inonu.edu.tr)
}

Received: Sep.2, 2021

Accepted: Oct.2, 2021

Published: Oct.20, 2021

\begin{abstract}
Analysis of brain activities and remote control are among the current issues that are being studied. Analysis of signals arising during brain functions is electroencephalography (EEG). EEG signals have intellectual, visual stimulation, and motion resultant forms. Especially, EEG signals generated by visual stimulus are within the scope of this study. In this study, research was carried out on the classification of EEG signals formed in a person looking at visual figures. For these studies, first of all, EEG signals from the brain were recorded with images and filtered to remove noise. Then, the features were extracted from the signals. In this study, Moment 5 feature was also used in addition to the features used in many studies such as mean, median, standard deviation and entropy. Then, classification was made using Support Vector Machine (SVM), k Nearest Neighbor (KNN), and Decision Tree (DT) algorithms. Classification was made for 4 different visual shapes used, since these shapes are square, circle, triangle, and star, and the same categorical names were used in the classification stage. As a result of the classification of EEG signals; SVM and KNN algorithms have determined which shape is viewed with $99.99 \%$ accuracy. These results show that different signals are produced in the brain according to the structure of the shape viewed. This situation shows that it can be used as a method to give patients the opportunity to express their requests just by looking or thinking.
\end{abstract}

Keywords: EEG, brain signal, visual stimulus, shape prediction.

\section{Introduction}

The basis of current studies on the brain computer interface in the field of control and command is based on the classification and interpretation of biomedical signals such as EEG. EEG signals are both stimulus and thought-based electrical potentials that occur during brain activity (Hu and Zhang 2019).

\subsection{Motivation}

Many studies address the classification of EEG signals by considering different methods and qualities. Many processes are tried to be performed with intellectual signals and visual stimulating signals. In particular, the issue of disease detection and control with brain signals has become popular topics. In this case, in order to use the EEG signals for the benefit of humans, the accuracy rate should be made with minimum error when classifying. 


\subsection{Problem Statement}

In this study, it was investigated on the classification of EEG signals using visual stimuli. For this purpose, 4 different black and white visuals were created which are circle, square, star and triangle images. The things to be done for this stage are listed as follows.

- Recording of signals

- Pre-processing of signals

- Feature extraction

- Classification of the feature

\subsection{Proposed Approach}

EEG signals should be classified and interpreted within the scope of this study. In order to do these operations, 4 black and white visuals were generated at the first step. Shapes are circles, squares, stars, and triangles. Later, the person was faced to observe these shapes on a screen where EEG signals will be received. At this stage, EEG signals were recorded every 1 second. For this purpose, an EEG device with 32 electrodes was used. The process of placing the electrodes of the device on the skull was done with the internationally accepted 10/20 system. In order for this placement to be performed flawlessly, the electrodes were placed in their sockets on it using the EPOC Flex Cap suitable for this system, and was placed on the skull after the gel is applied.

The contact and transmission of the electrodes were checked, and the recording phase was begun when the person standing still, starts to see the images on the screen. The map showing electrode placement and contact quality is given in Figure 1.

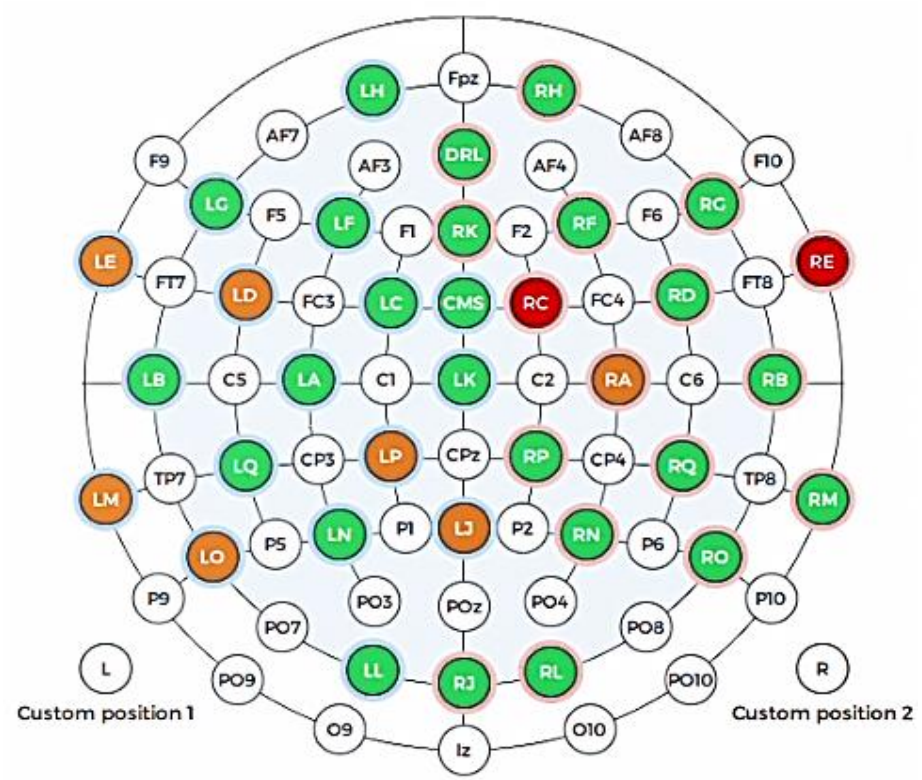

Figure 1. Electrodes placed according to the $10 / 20$ system and their data transmission qualities.

During the recording phase, each figure was displayed separately, and the signals for the displayed figure was recorded. The feature of the object shown in the potential formed in the brain of the person exposed to visual stimulus was tried to be determined in the next stages. In order to remove noise and artifacts, the recorded signals were first passed through a band-pass filter. The used band-pass filter passed signals between $0.5 \mathrm{hz}$ and $50 \mathrm{hz}$ bands. Due to the structure of the dataset generated, the electrode data of each 1 second signal were added one after the other and converted into a signal vector. These steps were performed and labeled separately for each shape. Thus, which signal belongs to which shape is determined in the dataset. At the end of this process, the dataset is ready for knowledge acquisition. 
After the signals were preprocessed as desired, feature extraction operations were performed. The features are Mean, Median, Standard Deviation, Moment 5 and Entropy. The feature vector of signal obtained for each shape was generated, and these feature vectors were stored for classification stage.

The last thing to do is to classify these features according to the determined methods such as SVM, KNN and DT.

\subsection{Related Work}

Many studies have been conducted on EEG signals from past to present. Some of these studies were conducted on EEG signals resulting from visual stimuli, disease symptoms, human movements and thoughts. Some of the studies at this stage are as follow. Lotte et al. (Lotte et al. 2018), investigated the methods used on 10-year EEG classification in a review study. Classifiers used in this field in their research; They found that adaptive classifiers, matrix and tensor classifiers, transfer learning and deep learning classifiers exist. Among these methods, they have shown that adaptive classifiers are generally superior to static ones even in unsupervised adaptations. Jiang et al. (Jiang et al. 2019), examined the studies on the removal of artifacts in EEG signals in a review study, and some methods such as Blind Source Separation (BSS) or Wavelet remove artifacts with great accuracy, but they found that online applications of these methods working with high computational complexity would not be suitable. Sazgar et al. (Sazgar and Young 2019),, have provided a detailed resource on describing the symptoms and characteristics of epilepsy, and determining the structure of EEG signals. Studies on disease and addiction detection; Tuncer et al. (Tuncer et al. 2019), proposed a local scenario pattern (LSP) algorithm for the detection of epilepsy disease and they detected the disease with an accuracy rate of $93 \%$ using 256 features they obtained. Acharya et al. (Acharya et al. 2012), performed the classification of the signals received from the brains of alcoholic and normal individuals who were stimulated with visual stimuli, and they made the classification with an accuracy rate of 91.7\%. Singh et al. (Singh et al. 2020), obtained the Time-frequency (TF) domain properties from EEG signals with discrete wavelet transform (DWT), and proposed a CNN-based epilepsy detection algorithm and provided the detection of the disease with an accuracy rate of $97.74 \%$. San-Segundo et al. (San-Segundo et al. 2019), used Fourier, wavelet, and empirical mode decomposition for epileptic EEG data signals as input for deep neural network (DNN). They obtained an accuracy rate of $99.5 \%$ for the result of comparing epilepsy seizures with seizure free. Studies on visual stimulus; Namazi et al. (Namazi et al. 2016), reveal the flexibility of eye movements in relation to visual stimulus with complex fractal visuals using visual stimuli. They recorded and analyzed the EEG signals for 3 different fractal visual stimuli. As a result, they observed that images with higher complexity caused fixation eye movements with lower fractality. Chowdhury et al. (Chowdhury et al. 2020), presented a DNN-based approach to estimate reaction time (RT) using a single-attempt EEG representation in a visual stimulus-response experiment with 48 participants, achieving $94 \%$ accuracy for binary classification. Zheng et al. (Zheng et al. 2020), proposed a method for estimation by classifying the EEG signals from the Long Short Term Memory (LSTM) based EEG encoder and ResNet deep learning algorithm by matching the features, they extracted from the visuals shown to the person to be taken, and as a result, they made the classification with $90.16 \%$ accuracy. Martisius and Damasevicius (Martišius and Damaševičius 2016), conducted a study on playing graphic games with brain signals and used it for an online target shooting game implemented in the OpenViBE environment. They used wave atom transformation for the features and classified them with SVM with 80.5\% accuracy. Sreeja and Samanta (Sreeja and Samanta 2019), calculated wavelet energy from motor image data as a sparse feature extraction method in their study and created a dictionary. In their study, where they aimed to reduce the calculation time and keep the accuracy rate high by using a single feature, they classified the results with an average accuracy rate of $91.84 \%$ for 4 different classes of EEG data. Zhou et al. (Zhou et al. 2020), proposed a hybrid BCI with incorporation of SSVEP in EEG and blink-associated electrooculography (EOG) signals. They achieved an average accuracy rate of $95.42 \%$ during the classification of visuals corresponding to 12 characters. Shao et al. (Shao et al. 2020), aimed to prepaid the cleaning robot walking on the wall with the BCI they designed, with EEG signals. For their study, they classified the signals received from 7 healthy people with the canonical correlation 
analysis (CCA) algorithm and ensured the control and movement of the cleaning robot with an accuracy of $89.92 \%$. Murugappan et al. (Murugappan et al. 2009), studied the classification of EEG signals for 5 different emotions, which they recorded with the BCI system, which they created from five different healthy people in two different ways, namely visual and audio-visual. When the results obtained in their studies are evaluated, the result obtained by using the audio-visual stimuli together is better than the result obtained from only the visual stimulus, and gives a result that reaches an accuracy rate of $66.67 \%$. According to this result, it shows that using visual stimuli together with auditory stimuli are methods that can be recommended to improve the result in studies. Kapeller et al. (Kapeller et al. 2013), conducted a study on whether EEG signals obtained with steady-state VEP (SSVEP) or code modulated VEP (c-VEP) are effective in continuous control of robots. For this purpose, they recorded EEG signals from 11 healthy people to control the robot for 4 movements on the computer screen. As a result of their applications and evaluations, they determined that the c-VEP method gave better results both in terms of task completion time and accuracy rate calculation. Classification of EEG signals received with c-VEP was performed with $98.18 \%$ accuracy. Thus, they stated that the c-VEP method is more suitable for robot control than the SSVEP method. Studies on EEG signals received during sleep; Abdulla et al. (Abdulla et al. 2019), tried to identify the sleep phases by classifying the EEG signals by generating cohesion graphs and converting them into graph knots. In their study, they determined the sleep stages with $94 \%$ accuracy. Mousavi et al. (Mousavi et al. 2019), tried to determine the sleep stages of the signal by using a deep learning algorithm with a single channel approach without performing attribute and selection processes from EEG signals. They obtained an accuracy rate of over $90 \%$ for the stages from 2 to 6 . Diykh et al. (Diykh et al. 2020), extracted features from each window of the EEG signals using the sliding window method and classified these features by the least square SVM method. It is seen that the method they proposed is more successful than single-channel methods and the application stages are determined with an accuracy of $96.74 \%$. EEG studies on control; Karaduman and Karc1 (Karaduman and Karc1 2020), recorded EEG signals by adapting on the basis of eye and arm movements and suggested that the devices could be controlled by ensuring the correlation of detection of these movements. Faiz and Al-Hamadani (Faiz and Al-Hamadani 2019), extracted attributes from EEG signals using Autoregressive (AR) coefficients and Common Spatial Pattern (CSP) methods and ensured the control of the humanoid robot hand by classifying them. They ensured the control of the robot arm by obtaining an accuracy rate of $97.5 \%$ from the results they divided into 5 classes. Zgallai et al. (Zgallai et al. 2019), conducted a study on moving by classifying the EEG signals they recorded during their thoughts on moving left, right, forward and backward, the directions they determined for the control of the brain-controlled wheelchair they designed. The EEG signals analyzed for the application were recorded from 10 healthy people while they were thinking about the determined movements. They classified the received EEG signals with $96 \%$ accuracy and provided control of the wheelchair. Chen et al. (Chen et al. 2018), designed the 7-DOF robotic arm control with gaze-based SSVEP system with wireless EEG device. In their study, they processed and analyzed the EEG signals received from 12 healthy people for 4 seconds. They separated the 2-second part of the analyzed EEG signal for the visual stimulus and the other 2-second part for changing the gaze, and as a result, they achieved control with 92.78\% accuracy. Zhang et al. (Zhang et al. 2019), proposed the convolutional repetitive attention model (CRAM) method by which they could classify subject-independent EEG signals, and as a result of their studies, they correctly classified it with an accuracy rate of 59.1\%. Chatterjee et al. (Chatterjee et al. 2019), used the Adaptive Auto regressive (AAR) feature extraction technique from EEG signals in the Internet of Things (IoMT) environment, and proposed a fuzzy distinctibility matrix (FDM) -based technique for selection from these features. Finally, they classified the features they obtained and achieved $80 \%$ accuracy. Er et al. (Er et al. 2021), conducted a study on predicting the emotions people feel while listening to different types of music by recording EEG signals. They obtained spectrograms by windowing the EEG signals for each emotion they identified. In their study, they combined the deep features in the fully connected layer using the two known ready-trained deep learning architectures, AlexNet and VGG16, and then they made emotion estimation by classifying them. They determined 4 different emotions with a $73.8 \%$ accuracy rate. Kumar et al. (Kumar et al. 2017), proposed a frequency 
band selection method to obtain information. They stated that common spatial patterns are detected in overlapping bands. LDA is applied to each filter properties. The scores obtained were combined and classified by SVM. They stated that they got better results than other studies that classified them with the lowest error value.

\subsection{Contribution}

In this study, it investigated whether the signals generated by visual stimuli in the human brain are different or not. For this purpose, EEG signals were recorded while the visuals of 4 different shapes are generated and the visuals were viewed, and then their features were extracted and classified. It is possible to determine which visual stimulation of the person whose brain signals are analyzed by using the output obtained as a result of this study. Thus, this study aims to add value to science and human life in order to ensure that individuals who cannot move due to problems in the skeletal and muscular system ask for their needs by looking at visuals or thinking about the visuals of what they want. In this respect, contributing to the field of science is among the aims of this study.

\section{System Theory}

In this study, EEG signals were recorded and classified using visual stimuli. The general flow diagram of the system proposed for this purpose is given in Figure 2.

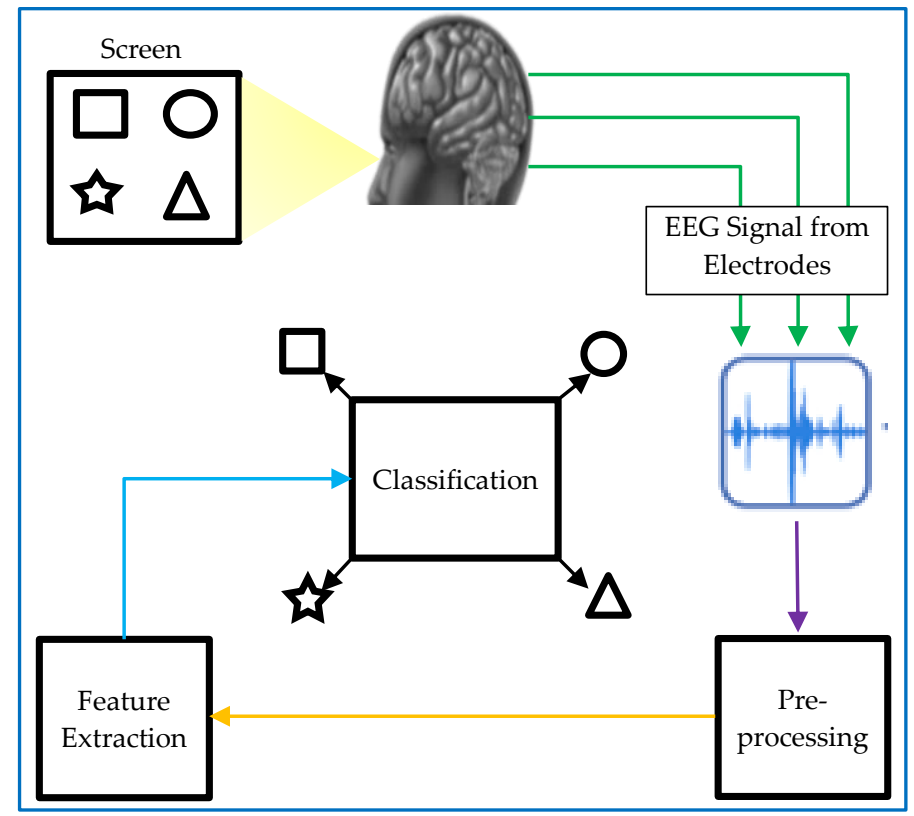

Figure 2. Shape classification from EEG signal system diagram.

In the proposed system, firstly the electrodes from which the EEG signals will be received were placed on the subject's skull with a 10/20 system by applying gel. For this process, electrodes were placed in the holes drilled on the cap to place the electrodes in the right places and attached to the skull. After this stage, the data transmission of the electrodes was checked first. When correct contact and data transmission are provided, a subject in a calm state goes across the screen and the recording of the signals begins. The steps followed to perform signal recording and other transactions are shown in Figure 3.

In Figure 3, firstly, training is carried out from the dataset constructed to generate a dataset and determine the classes. In the test steps, it is decided which shape the recorded signal belongs to. 4 different shapes were shown on the screen at different moments. Square, circle, triangle and star shapes were used for this process. White shape color and black background color were used for each shape. The recording starts with the displaying of the figure and the recording is released in the dataset as 1 second signals at $128 \mathrm{hz}$ frequency. Thus, separate records were made for each figure and the signals were recorded. 


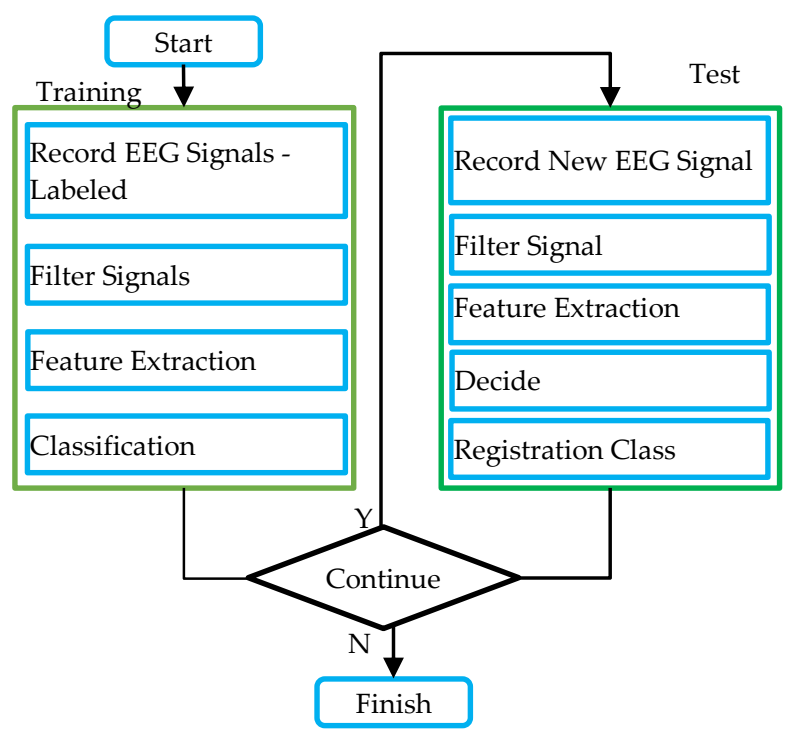

Figure 3. Step-by-step representation scheme for the application.

After this process, the signals are filtered. In the filtering stage, a band-pass filter that passes signals between $0.50 \mathrm{hz}$ and $50 \mathrm{hz}$ was used, and it was transformed into a clean signal by removing the signals considered as noise other than the EEG signal. The band-pass filter is designed as in Figure 4.

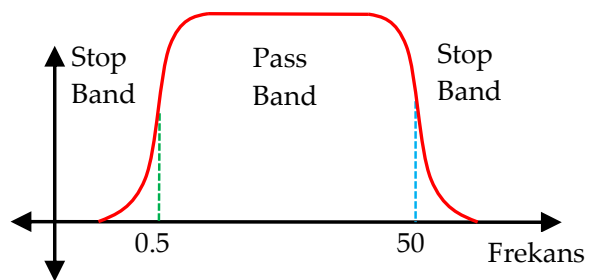

Figure 4. Band-pass filter design band graphic.

After the preliminary processes were completed, the dataset was on hand to release the classification process. At this stage, feature extraction stage has been conducted. The visual structure of the generated data is given in Figure 5. In Figure 5, V represents the generated signal vectors and E values represent the signals received from the electrodes.

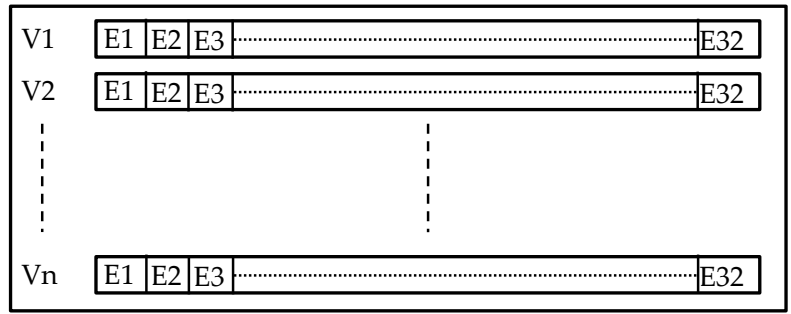

Figure 5. Recording of the created EEG shape data.

\subsection{Calculation of Features}

While extracting the features, 5 different features were determined. These features are mean, median, standard deviation, moment 5 and entropy. While calculating the average feature, calculations were made for every 1 second signal. When calculating, all electrical potential values contained in the signal for 1 second were summed up and calculated by dividing by the number of values in the signal. There are 4096 values in each signal vector. The other feature is the media. While calculating the median feature of the signal, all values in the signal were sorted in ascending order and the value in the middle is calculated as the median. The other feature is the media. While calculating the median feature of the signal, all values in the signal were sorted in ascending order and the value in the middle is calculated as the median. Another feature is the standard deviation. It is equal to the square root of the sum of the 
squares of the difference of the value of each 1 second signal from the mean. Its calculation is performed by equation 1 .

$$
\sigma=\sqrt{\frac{1}{n} \sum_{k=1}^{n}\left(x_{k}-\mathrm{a}\right)^{2}}
$$

In Equation 1, $\sigma$ indicates the standard deviation, a mean, the number of samples in $\mathrm{n}$ signals, $\mathrm{x}$ the value of the sample in the signal, and $\mathrm{k}$ the sample number.

$$
m_{r}=\frac{\sum_{i=1}^{N}\left(x_{i}-a\right)^{r}}{N}
$$

$\mathrm{mr}$ corresponds to any mean value of the distribution in any data. The moment is calculated by equation 2. Taking the $\mathrm{r}$ value as 5, the 5th moment is calculated and used as a feature (Kilicoglu, 2020).

$$
H_{p}=-\sum_{i=1}^{n} p_{i} \log _{2}\left(p_{i}\right)
$$

Finally, it is used as an entropy feature. Entropy is known as the amount of different information of the system and is defined as a measure of the sum of the thermal energy of the system per unit temperature (Karc1, 2016). Entropy is calculated by equation 3 (Ellerman, 2013).

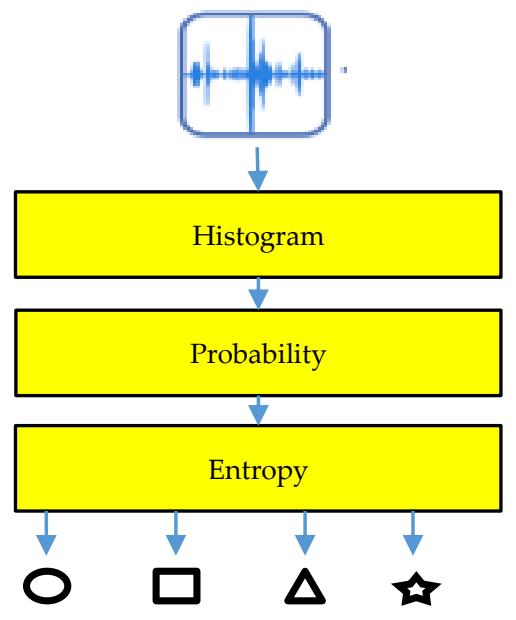

Figure 6. Entropy calculation steps for the EEG signals of the figures.

The steps of obtaining the entropy feature are as in Figure 6. In order to calculate entropy, the histogram of the signal is calculated first. The histogram graph for the calculated sample signal is given in Figure 7.

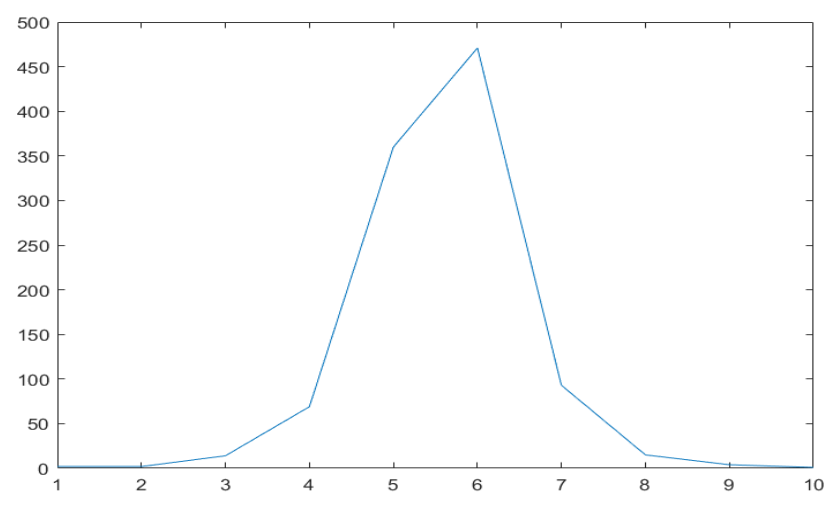

Figure 7. Sample histogram plot for EEG signal recorded from triangle shape.

Then probabilities were calculated for each histogram value. After probabilities are calculated, Entropy was calculated by using equation 3 . After the features were classified, the sample was recorded as numerical feature vectors as in Table 1 and the second stage of the dataset is completed by writing the shape they belong to. 
Table 1. Vector examples of feature values.

\begin{tabular}{|l|l|l|l|l|l|l|}
\hline Vector & Mean & Median & Standard Deviation & Moment 5 & Entropy & Shape \\
\hline V1 & 0.04 & -0.61 & 30.14 & $3.59 \times 10^{8}$ & $-8.44 \times 10^{6}$ & Circle \\
\hline V2 & -6.05 & -0.078 & 46.38 & $-5.48 \times 10^{10}$ & $-2.28 \times 10^{7}$ & Square \\
\hline V3 & -3.77 & -0.17 & 55.44 & $8.39 \times 10^{10}$ & $-3.12 \times 10^{7}$ & Triangle \\
\hline V4 & 2.65 & 0.37 & 24.26 & $9.41 \times 10^{8}$ & $-5.59 \times 10^{6}$ & Star \\
\hline V5 & -0.10 & -0.34 & 30.50 & $3.85 \times 10^{8}$ & $-8.67 \times 10^{6}$ & Circle \\
\hline
\end{tabular}

\subsection{Feature Classification}

At this stage, the values in the feature vector were classified using traditional classification methods such as SVM, KNN and DT. They are supervised learning methods used for SVM, classification, regression and outlier detection. In general classification process, an input field represented by $\mathrm{X}=(\mathrm{x} 1$, $\mathrm{x} 2, . ., \mathrm{xn}$ ) is classified into output space represented by $\mathrm{C} 1, \mathrm{C} 2, \ldots . . ., \mathrm{Ck}$. In classification, it is to match by finding the interconnection between $\mathrm{y}$ and $\mathrm{x}$ properties and to determine which class it belongs to (Çomak et al. 2007). To classify data, SVM tries to find the optimal separation sub-plane among all possible splitter planes. This classification process is shown as in Figure 8.

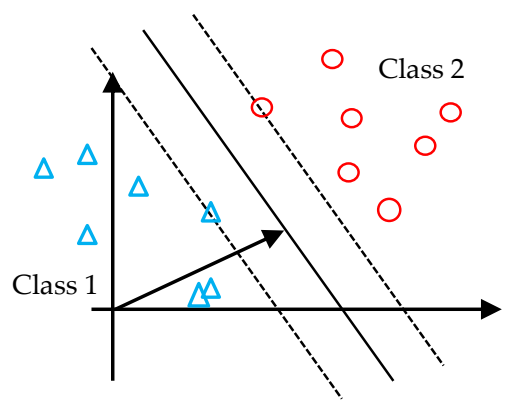

Figure 8. SVM simulation (Çomak et al. 2007).

The KNN algorithm, which is a supervised learning method used in the solution of classification and regression problems, can use Ibn-i Sina (Euclidean), Manhattan or Minkowski distances while calculating distances. In this study, Ibn-i Sina distance is used to calculate the distance. Ibn-i Sina distance and distance values between each vector were calculated and it was determined which value would be in which class. Example classification visual for $\mathrm{k}=3$ value is shown in Figure 9. As a result of the calculated distances $\mathrm{d}$, it is determined in which class the new value will be included (Çomak et al. 2007).

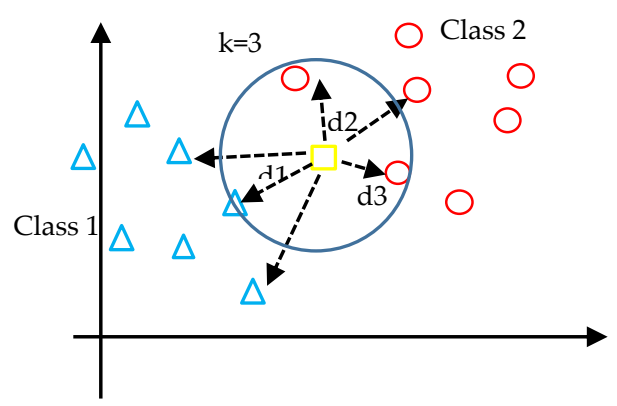

Figure 9. KNN simulation representation (Çomak et al. 2007).

Tree-based methods, one of the supervised learning algorithms, are frequently used in classification and regression problems. They have a structure that descends from the top. Entropy, Gini and least squares method algorithms are used to descend to sub-nodes. By looking at the value of the Gini index, it is first decided from which attribute it can be divided. This Gini value shows the proportion of 
variables in the data set (Adak and Yurtay 2017). The Gini value is obtained after calculating the right and left Gini values. Equations 4 and 5 show how right and left Gini values are calculated.

$$
\begin{aligned}
& \text { Gini }_{l}=1-\sum_{k=1}^{n}\left(\frac{L_{k}}{T_{l}}\right)^{2} \\
& \text { Gini }_{r}=1-\sum_{k=1}^{n}\left(\frac{R_{k}}{T_{r}}\right)^{2}
\end{aligned}
$$

In this equation, $\mathrm{n}$ is the number of classes, $\mathrm{T}$ is the number of samples in the node, $\mathrm{T}_{1}$ is the number of samples on the left arm, $T_{r}$ is the number of samples on the right arm, $\mathrm{Lk}$ is the number of samples in the $k$ category on the left arm, $R_{k}$ is the number of samples in the k category on the right arm (Adak and Yurtay 2017). After the calculations are made, the Gini value is calculated with equation 6 . Calculation is made for each attribute and the division is made on the attribute with the smallest value.

$$
\text { Gini }_{j}=\frac{1}{n}\left(T_{l} \text { Gini }_{l}+T_{r}\right)
$$

As a result of the classification methods, it is decided which class it belongs to and its accuracy rates are calculated.

\section{Experimental Result}

The data used in this study were recorded using Emotiv EPOC Flex. The images in Figure 10 are the ones used when recording EEG signals.
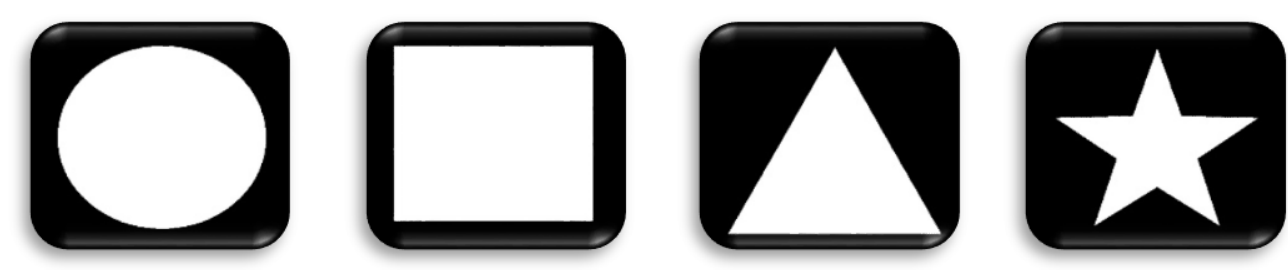

Figure 10. Shape images used for EEG signals.

Each visual was shown to the subject on a separate screen in full screen and EEG signals were recorded. EEG signals were recorded from 32 channels at $128 \mathrm{hz}$. During the recording phase, 1 second records were made and a dataset was generated. Every 1 second data were filtered to be free from noise, and a band-pass filter designed at this subject. At this stage, data belonging to signals between $0.5 \mathrm{hz}$ and $50 \mathrm{hz}$ frequency range are now included.
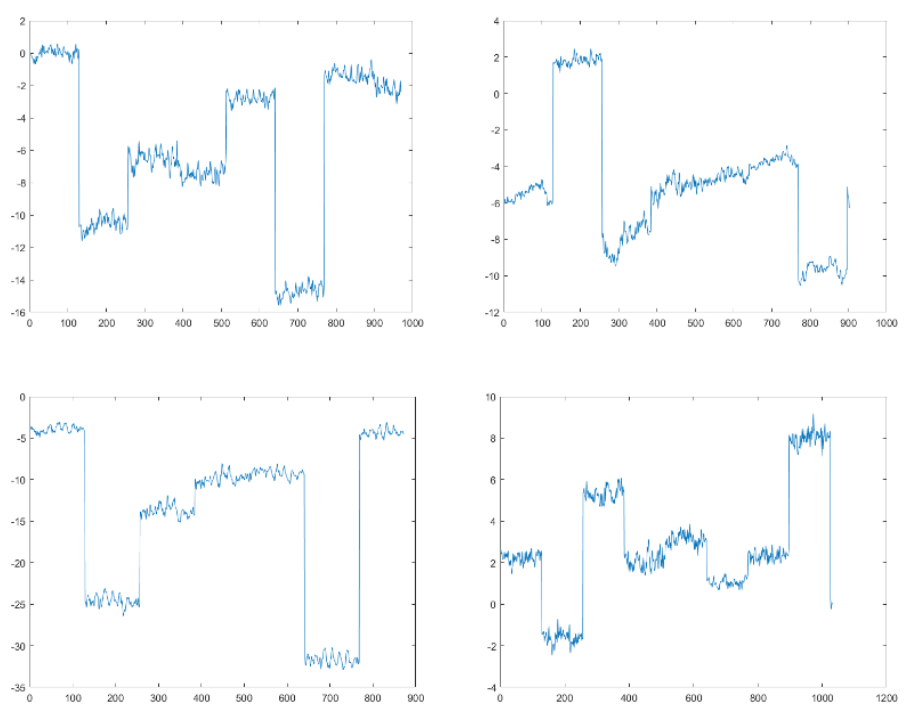

Figure 11. Mean feature results for EEG data of Circle, Square, Triangle and Star shapes.

Now the feature extraction phase was started. First of all, calculations were made for the mean feature. The calculations made are graphically shown in Figure 11. The graphs in Figure 11 are the 
graphical representation of the average values calculated from all signals in the data of square, circle, triangle and star shapes.

Median calculation was made as the other feature. After the median calculation was made separately for all signals in the data set, the obtained values were stored. The graphical results of the recorded values calculated for each visual are given in Figure 12.
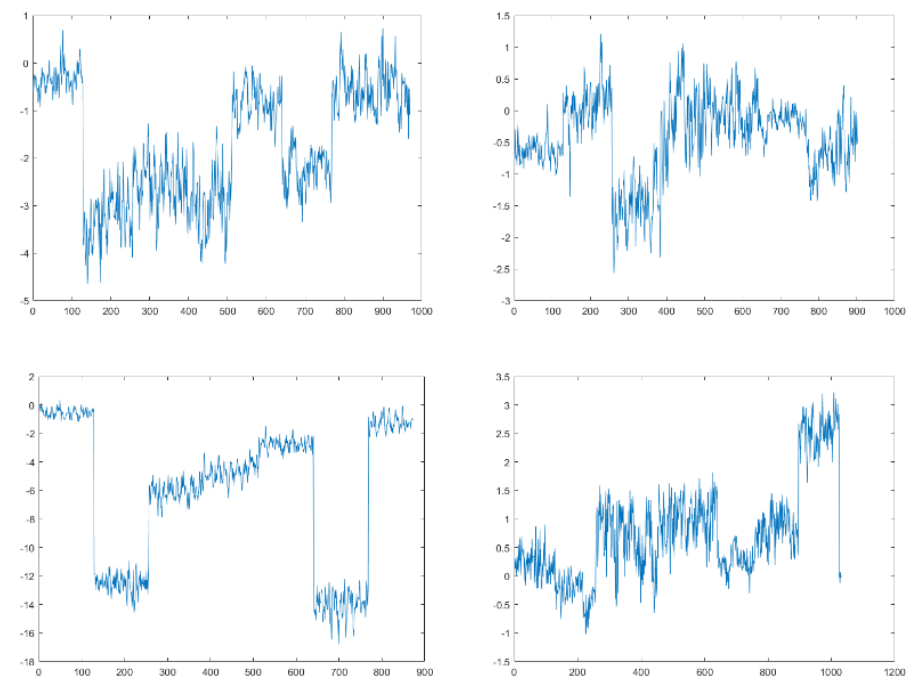

Figure 12. Median feature results for EEG data of Circle, Square, Triangle and Star shapes.

The calculation results were made separately from the signals recorded for each shape in the standard deviation feature given in the graphics in Figure 13.
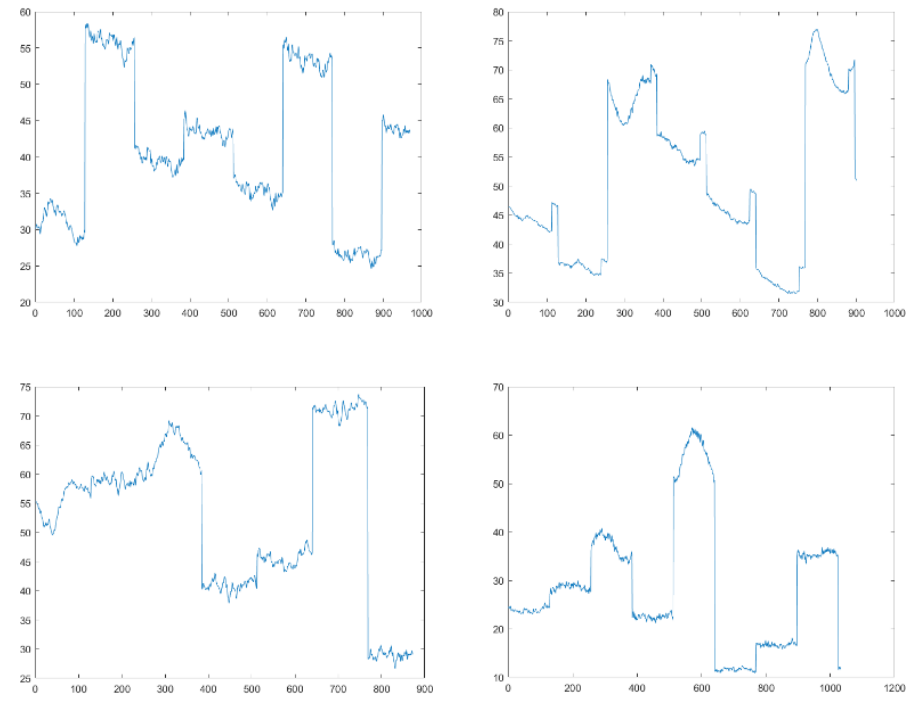

Figure 13. Standard deviation feature results for EEG data of Circle, Square, Triangle and Star shapes. 

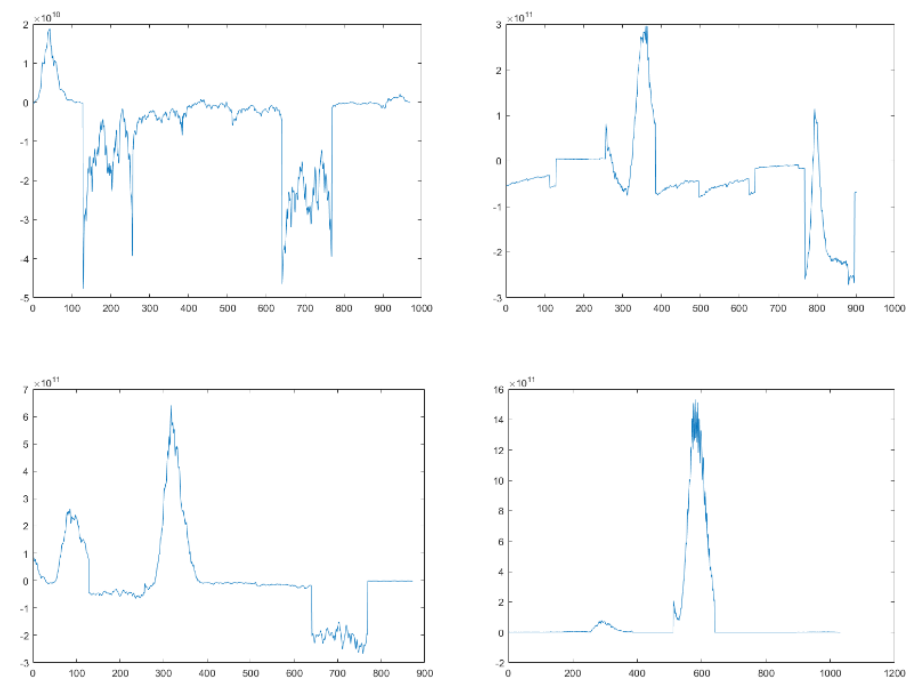

Figure 14. Moment 5 feature results for EEG data of Circle, Square, Triangle and Star shapes.

Similarly, the graphs of the results obtained as a result of the calculations for moment 5 , another feature, are given in Figure 14, and the graphs of the results obtained as a result of the calculations for entropy are also given in Figure 15.
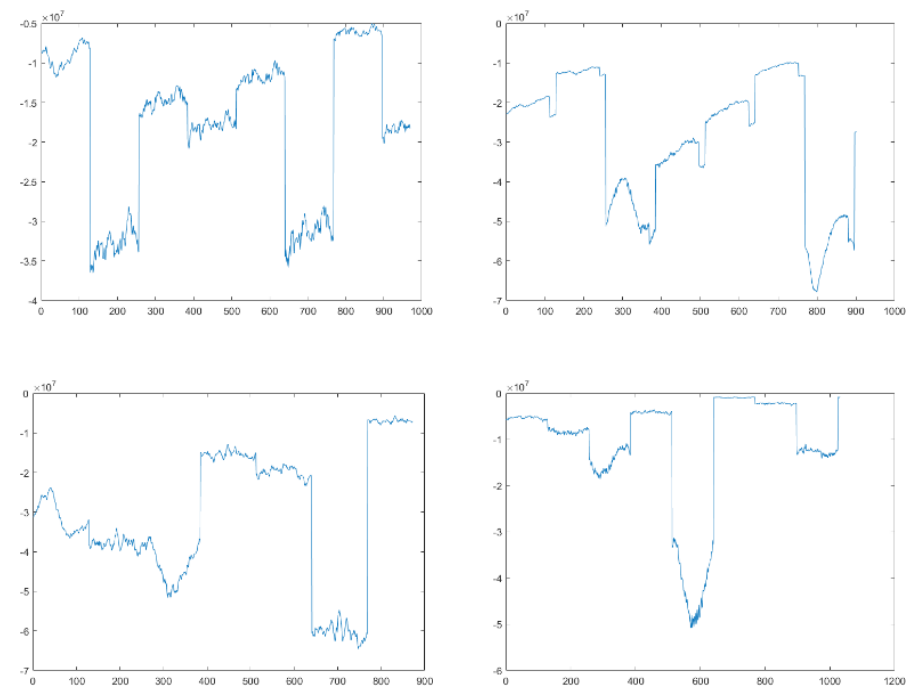

Figure 15. Entropy feature results for EEG data of Circle, Square, Triangle and Star shapes.

After the calculation of the features is completed, the step of classifying these 5 features is started. At this stage, the education stage is started in order to determine the classes. After determining which class belongs to the feature vector for each class, the classification process is performed with SVM, KNN and DT.

First of all, each feature is evaluated separately by using it in the classification process of figures. Classification results made separately are included in Table 2 . When the table is examined, it is seen that the best classification results are obtained for the mean feature when separate evaluations are made. The second place is the results obtained with the Moment 5 feature. 
Table 2. Classification of signals according to individual features.

\begin{tabular}{|l|l|l|l|l|l|}
\hline Method & Mean & Median & Standard Deviation & Moment 5 & Entropy \\
\hline SVM & $75.5 \%$ & $65.9 \%$ & $60.0 \%$ & $60.1 \%$ & $60.7 \%$ \\
\hline KNN & $76.8 \%$ & $65.5 \%$ & $59.9 \%$ & $70.6 \%$ & $62.7 \%$ \\
\hline DT & $74.8 \%$ & $65.3 \%$ & $60.5 \%$ & $70.1 \%$ & $60.8 \%$ \\
\hline
\end{tabular}

After this stage, the classification of the signals belonging to the shapes was carried out by using the features (Square-Circle, Square-Triangle, etc.) in combination. The classification results obtained on the figures in binary combinations are given in Table 3.

Table 3. Classifier accuracy rates for shape data in combinations of two.

\begin{tabular}{|l|c|c|c|}
\hline \multicolumn{1}{|c|}{ Shapes } & DT & SVM & KNN \\
\hline Square-Circle & $99.2 \%$ & $100 \%$ & $100 \%$ \\
\hline Square-Triangle & $98.8 \%$ & $99.9 \%$ & $100 \%$ \\
\hline Square-Star & $99.8 \%$ & $100 \%$ & $100 \%$ \\
\hline Circle-Triangle & $99.3 \%$ & $99.9 \%$ & $99.9 \%$ \\
\hline Circle-Star & $99.9 \%$ & $100 \%$ & $99.9 \%$ \\
\hline Triangle-Star & $100.0 \%$ & $100 \%$ & $100 \%$ \\
\hline
\end{tabular}

When the results in Table 3, where the classifications are obtained by comparing them in binary combinations, are examined, SVM and KNN give an accuracy rate of $100 \%$ for the Square-Circle combination. For Square-Triangle combination, KNN has 100\% accuracy. The Square-Star combination has an accuracy rate of $100 \%$ with SVM and KNN. The circle-star combination is classified by SVM with $100 \%$ approval. Triangle-Star combination, on the other hand, yielded $100 \%$ accuracy in all classification algorithms used. At the last stage of the study, the performance ratio of the signals of all shapes will be examined at the same time and by using all of the features. At this stage, the features obtained from the data of all signals are classified using KNN, SVM, DT algorithms. The results of these classifications are given in Table 4.

Table 4. Classification results of EEG signals.

\begin{tabular}{|c|c|}
\hline Classifier & Accuracy (\%) (Square, Circle, Triangle, Star) \\
\hline SVM & 99.99 \\
KNN & 99.99 \\
\hline DT & 99.3 \\
\hline
\end{tabular}

When Table 4 is examined, it is seen that all shapes can be distinguished by EEG signals with $99.99 \%$ accuracy. This shows that the human brain reacts differently to different visual stimuli. The confusion matrix of the accuracy rate obtained as a result of the classification is given in Figure 16. The Star shape with only the number 3 has been incorrectly classified as the circle with the number 0 . Apart from this, all other signal data are classified correctly. It is given in Figure 17 in the graph with the ROC curve. 


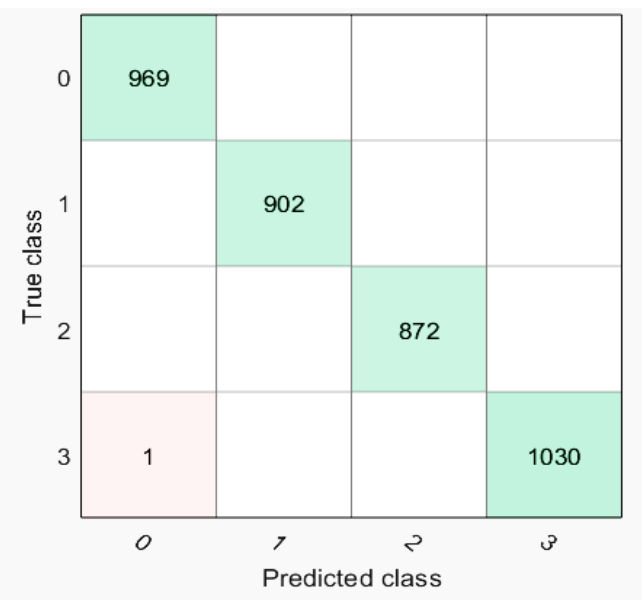

Figure 16. Confusion matrix for SVM classification of square, circle, triangle and star shapes by feature.

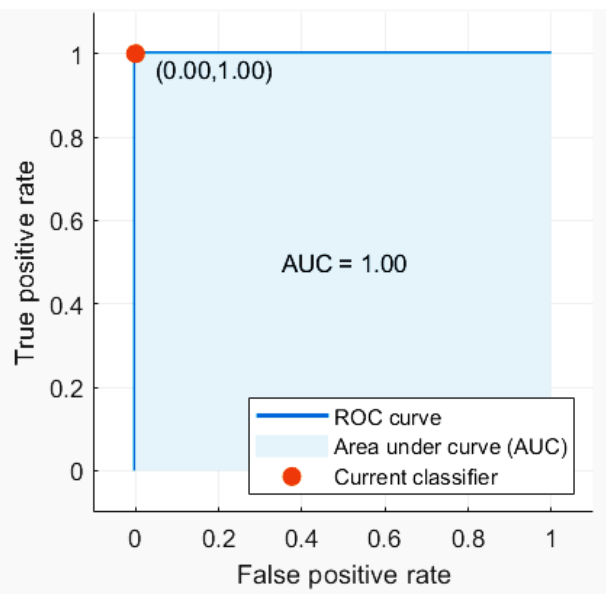

Figure 17. SVM ROC plot for prediction of square, circle, triangle and star shapes for feature.

Comparison with other studies in the literature using similar visual stimulus method is given in Table 5. When Table 5 is not analyzed, there are studies using different dataset and data. The majority of these studies are carried out on data recorded based on visual stimuli.

Table 5. Comparison with other studies in the literature.

\begin{tabular}{|l|l|l|}
\hline Reference & Method & Accuracy Rate \\
\hline (Acharya et al. 2012) & NNLS & $91.70 \%$ \\
\hline (Chowdhury et al. 2020) & DNN & $94.00 \%$ \\
\hline (Zheng et al. 2020) & ResNet & $90.16 \%$ \\
\hline (Martišius and Damaševičius 2016) & SVM & $80.50 \%$ \\
\hline (Zhou et al. 2020) & CCA & $95.42 \%$ \\
\hline (Shao et al. 2020) & CCA & $89.92 \%$ \\
\hline (Murugappan et al. 2009) & NN & $66.67 \%$ \\
\hline (Kapeller et al. 2013) & LDA & $98.18 \%$ \\
\hline Proposed Method & SVM & $99.99 \%$ \\
& KNN & $99.99 \%$ \\
\hline
\end{tabular}

\section{Acknowledge}

This authors would like to thank to Scientific Research Project Coordination Unit at Inonu University due to their support in granted project FBA-2019-1664. 


\section{Conclusion}

In this study, EEG signals received from a person looking at the visual were analyzed. The purpose of the analysis process is to estimate the visual image with EEG signals. For this purpose, 4 different shapes were determined and EEG signals were recorded with a 32-channel device by looking at these shapes. After the preprocessing step and feature extraction step, the classification phase was started and as a result, the classification success was achieved with $99.99 \%$ accuracy. A basic problem of sick people was tried to be solved with the success of proposed method in this study. This problem is the automatic determination and fulfillment of the requests of the patients who have lost the ability to move, by looking at the images, with the EEG signals. With the development of real systems similar to the structure of this study, convenience will be provided for both patients and those who take care of them.

In future studies, studies will be carried out to determine the shapes in the picture by looking at different pictures containing geometric shapes, and to determine the location of the shape by determining the eye directions from the EEG signals. In addition, studies will be carried out on whether EEG signals are discriminatory for different colors.

\section{References}

Abdulla S, Diykh M, Laft RL, Saleh K, Deo RC (2019) Sleep EEG signal analysis based on correlation graph similarity coupled with an ensemble extreme machine learning algorithm. Expert Syst Appl 138:112790 . https://doi.org/10.1016/j.eswa.2019.07.007

Acharya UR, Sree SV, Chattopadhyay S, Suri JS (2012) Automated diagnosis of normal and alcoholic EEG signals. Int J Neural Syst 22: . https://doi.org/10.1142/S0129065712500116

Adak MF, Yurtay N (2017) Gini Algoritmasını Kullanarak Karar Ağacı Oluşturmayı Sağlayan Bir Yazılımın Geliştirilmesi Developing a Software Which Provides Creating Decision Trees by Using Gini Algorithm. 1-6

Chatterjee R, Maitra T, Hafizul Islam SK, Hassan MM, Alamri A, Fortino G (2019) A novel machine learning based feature selection for motor imagery EEG signal classification in Internet of medical things environment. Futur Gener Comput Syst 98:419-434 https://doi.org/10.1016/j.future.2019.01.048

Chen X, Zhao B, Wang Y, Xu S, Gao X (2018) Control of a 7-DOF Robotic Arm System with an SSVEPBased BCI. Int J Neural Syst 28: . https://doi.org/10.1142/S0129065718500181

Chowdhury MSN, Dutta A, Robison MK, Blais C, Brewer GA, Bliss DW (2020) Deep neural network for visual stimulus-based reaction time estimation using the periodogram of single-trial eeg. Sensors (Switzerland) 20:1-20 . https://doi.org/10.3390/s20216090

Çomak E, Arslan A, Türkoğlu I (2007) A decision support system based on support vector machines for diagnosis of the heart valve diseases. Comput Biol Med 37:21-27 . https://doi.org/10.1016/j.compbiomed.2005.11.002

Diykh M, Li Y, Abdulla S (2020) EEG sleep stages identification based on weighted undirected complex networks. Comput Methods Programs Biomed 184: . https://doi.org/10.1016/j.cmpb.2019.105116

Ellerman D (2013) An introduction to logical entropy and its relation to shannon entropy. Int J Semant Comput 7:121-145 . https://doi.org/10.1142/S1793351X13400059

Er MB, Çiğ H, Aydilek İB (2021) A new approach to recognition of human emotions using brain signals and music stimuli. Appl Acoust 175: . https://doi.org/10.1016/j.apacoust.2020.107840

Faiz MZ Al, Al-Hamadani AA (2019) Online brain computer interface based five classes EEG to control humanoid robotic hand. 2019 42nd Int Conf Telecommun Signal Process TSP 2019 406-410 . https://doi.org/10.1109/TSP.2019.8769072

Hu L, Zhang Z (2019) EEG Signal Processing and Feature Extraction. Springer Singapore, Singapore 
Jiang X, Bian G Bin, Tian Z (2019) Removal of artifacts from EEG signals: A review. Sensors (Switzerland) 19:1-18 . https://doi.org/10.3390/s19050987

Kapeller C, Hintermuller C, Abu-Alqumsan M, Pruckl R, Peer A, Guger C (2013) A BCI using VEP for continuous control of a mobile robot. Proc Annu Int Conf IEEE Eng Med Biol Soc EMBS 5254-5257 . https://doi.org/10.1109/EMBC.2013.6610734

Karaduman M, Karc1 A (2020) Controlling Vehicles Using EEG Signal And Eye-Arm Collaboration. In: ISERD 180th International Conference. Macca, pp 19-24

Karc1 A (2016) Fractional order entropy: New perspectives. Optik (Stuttg) 127:9172-9177 . https://doi.org/10.1016/j.ijleo.2016.06.119

Kilicoglu

$\mathrm{T}$.

Astroistatistik, https://acikders.ankara.edu.tr/pluginfile.php/107751/mod_resource/content/0/Astroistatistik_Ko nu_05_Momentler_Carpiklik_ve_Basiklik.pdf (15.12.2020)

Kumar S, Sharma A, Tsunoda T (2017) An improved discriminative filter bank selection approach for motor imagery EEG signal classification using mutual information. BMC Bioinformatics 18: . https://doi.org/10.1186/s12859-017-1964-6

Lotte F, Bougrain L, Cichocki A, Clerc M, Congedo M, Rakotomamonjy A, Yger F (2018) A review of classification algorithms for EEG-based brain-computer interfaces: A 10 year update. J Neural Eng 15: . https://doi.org/10.1088/1741-2552/aab2f2

Martišius I, Damaševičius R (2016) A prototype SSVEP based real time BCI gaming system. Comput Intell Neurosci 2016: . https://doi.org/10.1155/2016/3861425

Mousavi Z, Yousefi Rezaii T, Sheykhivand S, Farzamnia A, Razavi SN (2019) Deep convolutional neural network for classification of sleep stages from single-channel EEG signals. J Neurosci Methods 324: . https://doi.org/10.1016/j.jneumeth.2019.108312

Murugappan M, Juhari MRBM, Nagarajan R, Yaacob S (2009) An investigation on visual and audiovisual stimulus based emotion recognition using EEG. Int J Med Eng Inform 1:342-356 . https://doi.org/10.1504/IJMEI.2009.022645

Namazi H, Kulish V V., Akrami A (2016) The analysis of the influence of fractal structure of stimuli on fractal dynamics in fixational eye movements and EEG signal. Sci Rep 6:1-8 . https://doi.org/10.1038/srep26639

San-Segundo R, Gil-Martín M, D’Haro-Enríquez LF, Pardo JM (2019) Classification of epileptic EEG recordings using signal transforms and convolutional neural networks. Comput Biol Med 109:148-158 . https://doi.org/10.1016/j.compbiomed.2019.04.031

Sazgar M, Young MG (2019) Absolute Epilepsy and EEG Rotation Review: Essentials for trainees

Shao L, Zhang L, Belkacem AN, Zhang Y, Chen X, Li J, Liu H, Minati L (2020) EEG-Controlled WallCrawling Cleaning Robot Using SSVEP-Based Brain-Computer Interface. J Healthc Eng 2020: . https://doi.org/10.1155/2020/6968713

Singh A, Pusarla N, Sharma S, Kumar T (2020) CNN-based Epilepsy detection using image like features of EEG signals. Int Conf Electr Electron Eng ICE3 2020 280-284 . https://doi.org/10.1109/ICE348803.2020.9122874

Sreeja SR, Samanta D (2019) Classification of multiclass motor imagery EEG signal using sparsity approach. Neurocomputing 368:133-145 . https://doi.org/10.1016/j.neucom.2019.08.037

Tuncer T, Dogan S, Akbal E (2019) A novel local senary pattern based epilepsy diagnosis system using EEG signals. Australas Phys Eng Sci Med 42:939-948 . https://doi.org/10.1007/s13246-019-00794-x

Zgallai W, Brown JT, Ibrahim A, Mahmood F, Mohammad K, Khalfan M, Mohammed M, Salem M, 
Hamood N (2019) Deep Learning AI Application to an EEG driven BCI Smart Wheelchair. 2019 Adv Sci Eng Technol Int Conf ASET 2019 14-18 . https://doi.org/10.1109/ICASET.2019.8714373

Zhang D, Yao L, Chen K, Monaghan J (2019) A Convolutional Recurrent Attention Model for SubjectIndependent EEG Signal Analysis. IEEE Signal Process Lett 26:715-719 . https://doi.org/10.1109/LSP.2019.2906824

Zheng X, Chen W, You Y, Jiang Y, Li M, Zhang T (2020) Ensemble deep learning for automated visual classification using EEG signals. Pattern Recognit 102:107147 . https://doi.org/10.1016/j.patcog.2019.107147

Zhou Y, He S, Huang Q, Li Y (2020) A Hybrid Asynchronous Brain-Computer Interface Combining SSVEP and EOG Signals. IEEE Trans Biomed Eng 67:2881-2892 https://doi.org/10.1109/TBME.2020.2972747 